\title{
Observing ultraviolet signatures of interstellar organics with the Hubble Space Telescope
}

\author{
Theodore P. Snow \\ Center for Astrophysics and Space Astronomy, University of Colorado \\ 389 UCB \\ Boulder, CO 80309-0389 \\ U.S.A. \\ e-mail: tsnow@casa.colordao.edu
}

\begin{abstract}
The Cosmic Origins Spectrograph (COS) will be more sensitive for ultraviolet spectroscopy than either the GHRS or the STIS, especially in the far UV where many absorption lines and bands formed by atoms and molecules have electronic transitions from the ground state. Here we outline our plans for using the COS to observe interstellar gas and dust in the cold ISM, along with a report on the results of preliminary archival HST search for UV diffuse interstellar bands.
\end{abstract}

Keywords. ISM: clouds, ISM: molecules, ultraviolet: ISM, astrobiology, space vehicles: instruments

\section{Preface}

At this writing, the Hubble Space Telescope (HST) was to be serviced by Space Shuttle astronauts in late October, 2008. By the date of this volume's publication, that servicing mission should have taken place. In this paper, we assume that the mission has been successful.

\section{Introduction to UV Observations of the ISM}

The value of making ultraviolet spectroscopic observations of the interstellar gas and dust was first conceptualized by Spitzer (1946), who envisioned a large telescope in space, to observe (among other things) interstellar UV absorption lines in the spectra of hot stars lying behind interstellar clouds. Spitzer's dream has been realized in the form of the Hubble Space Telescope.

In the very low densities of interstellar space, even in the densest clouds, collisions between particles are so rare that essentially all atoms and molecules remain in their ground electronic and vibrational state. In the energy-level structures of most universally abundant atoms and a number of common organic molecules, a wide gap exists between the ground and the first electronically excited states. Hence, most electronic transitions from the ground state lie at UV wavelengths. Despite the many challenges for would-be UV interstellar absorption-line observers, a lot has been learned about the diffuse ISM from UV observations, including the general composition of the gas (and hints about the composition of the dust), the physical and chemical conditions; and the kinetics of the gas and dust. But what is missing is detailed knowledge about denser interstellar clouds, because of the difficulties in making UV spectroscopic observations there, due to the high dust extinction. 
Our COS Science Team observing plan for the cold ISM is centered around UV spectra of stars seen behind translucent clouds. These clouds are defined by their total extinction (ranging between $A_{V} 1.5$ and $10 \mathrm{mag}$ ). Figure 1 shows, as a function of line-of-sight column density, the expected relative abundances of atomic and molecular hydrogen and carbon, of translucent clouds (see Snow \& McCall 2006 for references). The shaded area on the figure shows what parameter space $C O S$ can cover in observing absorption lines. Note that this figure, and the general definition of translucent clouds, refers to the local conditions in a single cloud, not to be confused with the average conditions integrated over the entire line of sight.

\section{The Capabilities of the Cosmic Origins Spectrograph}

The COS instrument and its capabilities are described in the paper by Green (2000), and on the Space Telescope Science Institute web site (www.stsci.edu/hst/cos). The emphasis in designing the instrument was placed on the far-UV, so a one-reflection, Rowlandcircle design was chosen for that spectral region (between $\sim 1,150$ and $\sim 1,800 \AA$ ), to achieve 10 to 20 times greater sensitivity than STIS at comparable spectral resolution. For the near-UV ( 2, 000 to $\sim 3,200 \AA)$ additional reflections are used, but the sensitivity to point sources is still greater than that of STIS. The COS has two resolution modes: the medium-resolution $(\mathrm{R} \sim 20,000)$ gratings, comparable to the M-gratings in STIS; and low-resolution gratings $(\mathrm{R} \sim 5,000)$.

\section{An Archival Search for Unidentified UV Absorption Features}

In starting to plan for our Guaranteed Time Observations, we (J. D. Destree and T. P. Snow) initiated an archival project using existing spectra obtained by STIS. This study led to some interesting results, which are outlined below.

\subsection{The detection of CS absorption in the $U V$}

In a blind search for unidentified absorption features, we found one near 1,400 , which we then able were able to identify as an absorption band of the diatomic molecule CS.

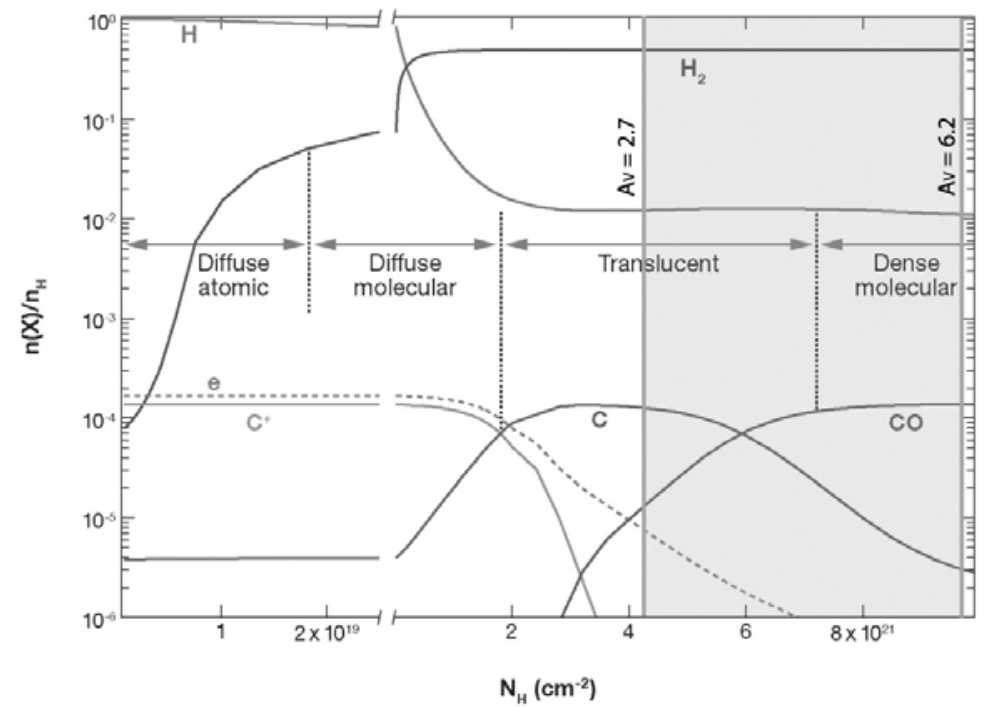

Figure 1. Relative abundance of atomic and molecular hydrogen and carbon of translucent clouds. The shaded area shows the parameter space the $C O S$ can explore and observe in absorption lines against hot background stars. 
This was the first verified detection of this molecule in the UV (but a detection had been tentatively made before; Smith et al. 2001). Of course, CS is commonly detected by radio observations in dark and translucent clouds (Scappini et al. 2007 and references cited therein), and theoretical models of dense and translucent clouds show that CS is formed easily by ion-neutral reactions (Drdla et al. 1989), so the UV detection was not a surprise when stars with high-enough reddening could be observed. We found CS in 16 out 21 lines of sight we checked; these results, including a correlation analysis, can be found in Destree et al. (2008).

\subsection{The search for diffuse interstellar bands}

The primary goal of our archival STIS project was to search for ultraviolet diffuse interstellar bands (i.e., UV DIBs), because that is a high-priority item in our COS GTO program, and we wanted to test the waters. The visible-wavelength DIBs have been known since the early 1920s (Heger 1922), but their carriers remain unidentified, almost 90 years later. After several false starts, now the consensus is that the DIBs are formed by a family of complex organic molecules. The paper by Peter Sarre in this volume elaborates on this point, as do previous reviews (e.g., Snow 2001).

Searches for UV DIBs have been made previously (Snow et al. 1977, Seab \& Snow 1985, Clayton et al. 2003, Jenkins et al. 2008), but no unequivocal DIBs were found. One UV DIB was detected in another study (Tripp et al. 1993), but that feature was later identified as CH (Sheffer \& Federman 2007 and references cited therein).

Our search, however, yielded several absorption features that conform to what we expect of UV DIBs: they are found only in at least moderately-reddened stars; they are truly interstellar; and they are unidentified. Further criteria were that any thought-tobe DIB must be observed in more than one line of sight; it must be broader than a typical interstellar atomic line while having a significantly different width than stellar lines; and where possible, we also tried find a feature in separate exposures on the same star (preferably with different grating settings, to rule out detector glitches). In our first attempt, we found ten or more features that survived all of our tests, and there are probably several more. The results of our archival search will be published by Destree \& Snow (2008).

\section{The COS Science Team's Observing Program on the Cold ISM}

As a member of the COS Science Team, the author is responsible for planning the Team's GTO observations of the cold ISM. Our adopted program is designed to explore the parameter space shown in the shaded region in Figure 1. We hope to measure column densities of atomic, ionic, and molecular gas-phase species in more heavily-reddened and denser lines of sight than previously possible to probe in the UV. The specific observing target list can be found on the internet, at www.stsci.edu/hst/proposing/docs/COSGTO/APTProposal_Snow.pdf.

As seen in Figure 1, much of the previously unexplored region represents translucent clouds. In these clouds, hydrogen becomes almost wholly molecular and carbon makes the transitions from $\mathrm{C}$ II to $\mathrm{C}$ I to $\mathrm{CO}$ (from $\mathrm{C}^{+}$to $\mathrm{C}$ to $\mathrm{CO}$, in terms used by chemists). While we can't observe $\mathrm{H}_{2}$, we can easily observe carbon in all its abundant forms, so we can trace carbon as it goes through the transitions we expect to see in these clouds.

Our scientific goals include: estimating elemental depletions (of gas-phase species onto dust) in translucent clouds, to see how the gas-dust interaction changes with increasing volume density and decreasing radiation field intensity; to test and improve chemical models of translucent clouds; to see whether and how the dust extinction curve is affected by the conditions in these clouds; and to search for new molecular species in translucent 
clouds, including species whose UV spectra are known, as well as searching for new unidentified species (i.e., UV DIBs). We will specifically search for PAHs, because that family is among the leading candidates for the carriers of the visible-wavelengths DIBs, and in their neutral form, many PAHs have electronic spectra in the UV. Some work has been done to measure the UV spectrum of these species in the lab (e.g., Salama et al. 1995, Salama 1999), so identifications should eventually be possible.

\section{HST Observations of Organics in Comets}

We (K. O'Malia and T.P. Snow) plan to carry out using HST absorption-line observations of cometary comae by stellar occultation measurements. When a comet passes in front of a hot star, we expect to see absorption lines caused by gas in the coma. This method has been tried in the past using visible light, without success (see Herbig \& McNally 1999) — but UV observations are a lot more likely to produce positive results, for all the same reasons that UV spectra are more productive than visible spectra for observing interstellar absorption lines. Despite the lack of success in previous absorption line searches, in a recent study (O'Malia et al. 2008), even visible-wavelength absorption lines were tentatively detected by comparing very high $\mathrm{S} / \mathrm{N}$ on-comet spectra with offcomet spectra of the same stars. Thus we are very hopeful that we will detect a host of atomic, ionic, and molecular species, including organics, when we apply UV spectroscopy to future comets.

\section{References}

Clayton, G. C., Gordon, K. D., Salama, F., Allamandola, L. J., Martin, P. G., Snow, T. P., Whittet, D. C. B., Witt, A. N., \& Wolff, M. J. 2003, ApJ, 592, 947

Destree, J. D. \& Snow, T. P. 2008, in preparation

Destree, J. D, Snow, T. P., \& Black, J. H. 2008, ApJ, submitted

Drdla, K., Knapp, G. R., \& Van Dishoeck, E. F. 1989, ApJ, 345, 815

Green, J. 2000, Proc. Soc. Photo-Opt. Eng., 4013, 352

Heger, M. L. 1922, Bull. Lick Obs., No. 337, 141

Herbig, G. H. \& McNally, D. 1999, MNRAS, 340, 951

Jenkins, E. B., Snow, T. P., \& Rachford, B. L. 2008, in preparation

O'Malia, K., Snow, T. P., York, D. G., Thorburn, J. D., et al., 2008, in preparation

Salama, F. 1999, in: L. d'Hendecourt, C. Joblin, \& A. Jones (eds.), Solid Interstellar Matter: The ISO Revolution, (New York: Springer), p. 65

Salama, F., Joblin, C., \& Allamandola, L. J. 1995, Planet. Space Sci., 43, 1165

Scappini, F., Cecchi-Pestellini, C., Casi, S., \& Olberg, M. 2007, A\&SA, 466, 243

Seab, C. G. \& Snow, T. P. 1985 ApJ, 295, 485

Sheffer, Y. \& Federman, S. R. 2007, ApJ, 659, 1352

Smith, A. M., Lyu, C.-H., \& Bruhweiler, F. C. 2001, Bull. AAS, 33, 1452

Snow, T. P. 2001, Spectrochimica Acta, Part A, 57, 615

Snow, T. P. \& McCall, B. J. 2006, ARAA, 44, 367

Snow, T. P., York, D. G., \& Resnick, M. 1977, PASP, 89, 758

Spitzer, L. 1946, Report To Project RAND: Astronomical Advantages of an Extra-Terrestrial Observatory (reprinted in Astr. Quarterly, 7, 131, 1990)

Tripp, T. M., Cardelli, J. A., \& Savage, B. D. 1994, AJ, 107, 645

\section{Discussion}

ZIURYS: This detection of CS is pretty interesting. You care to speculate what other diatomics may triatomics maybe detectable in the UV?

IRVINE: May be you are in a better position to tell me the answer to this question. 